\title{
Posterior tibial tendon displacement behind the tibia and its interposition in an irreducible isolated ankle dislocation: a case report and literature review
}

\author{
ALESSANDRO ORTOLANI ${ }^{1}$, ROBERTO BEVONI ${ }^{2}$, ALESSANDRO RUSSO ${ }^{1}$, \\ MAURILIO MARCACCI ${ }^{1}$, MAURO GIROLAMI ${ }^{2}$ \\ ${ }^{1}$ I Clinica Ortopedica, Istituto Ortopedico Rizzoli, Bologna, Italy \\ ${ }^{2}$ Ortopedia Bentivoglio, Istituto Ortopedico Rizzoli, Bentivoglio (BO), Italy
}

\begin{abstract}
Isolated posteromedial ankle dislocation is a rare condition thanks to the highly congruent anatomical configuration of the ankle mortise, in which the medial and lateral malleoli greatly reduce the rotational movement of the talus, and the strength of the ligaments higher than the malleoli affords protection against fractures. However, other factors, like medial malleolus hypoplasia, laxity of the ligaments, peroneal muscle weakness and previous ankle sprains, could predispose to pure dislocation. In the absence of such factors, only a complex high-energy trauma, with a rotational component, can lead to this event.

Irreducibility of an ankle dislocation, which is rarely encountered, can be due to soft tissue interposition. Dislocation of the posterior tibial tendon can be the cause of an irreducible talar dislocation; interposition of this tendon, found to have slid posteriorly to the distal tibia and then passed through the tibioperoneal syndesmosis, is reported in just a few cases of ankle fracture-dislocation.
\end{abstract}

Keywords: posterior tibial tendon, displacement, irreducible, ankle dislocation, open reduction.

\section{Introduction}

Irreducibility of an ankle fracture or fracture-dislocation is a rare occurrence that can be caused by soft tis-

\section{Corresponding Author:}

Alessandro Ortolani, MD

I Clinica Ortopedica

Istituto Ortopedico Rizzoli

Via di Barbiano 1/10, 40136 Bologna, Italy

E-mail: ortolanialessandro84@gmail.com sue interposition and/or entrapment of fracture fragments, dislocation of the fibula (anterior or posterior) in a tibiotalar fracture-dislocation, or interposition of the soft tissue-like deltoid ligament or posterior tibial tendon in a medial malleolar fracture. Another possible cause is entrapment of the extensor tendon in the distal tibiofibular joint by the extensor retinaculum in the presence of tibiotalar diastasis (1-5).

In the literature, dislocation of the posterior tibial tendon has been reported as the possible cause of irreducibility in a subtalar dislocation (6). Interposition of an anteriorly displaced posterior tibial tendon in a tibiotalar fracture-dislocation was first described by Böhler (7) in 1936; then came reports by Coonrad and Bugg (5) in 1954 and Parrish (8) in 1959; other cases were described in later years by different Authors (911). Further cases of posterior tibial tendon interposition between the distal tibia and fibula in tibiotalar fracture-dislocation have been reported in more recent years, but in these cases the tendon slid posteriorly to the distal tibia, passing through the disrupted tibioperoneal syndesmosis, and preventing reduction of the ankle fracture-dislocation (12-14).

To the Authors' knowledge, despite all these reported cases of ankle fracture-dislocation, the literature contains no reports of irreducible isolated (without fracture) ankle dislocation with posterior tibial tendon displacement, in which the tendon has slid posteriorly to the distal tibia and positioned itself in the tibiofibular interosseous space.

\section{Case report}

A 17-year-old man had a motor vehicle accident resulting in crush trauma to his right ankle; he also 
sustained abrasions to his right arm. When the patient arrived in our emergency room, exploration of the right ankle showed an axial deviation of the tibiotalar joint; his skin did not show any sign of impending ischemia, and no neurovascular deficit was present. Xrays showed an isolated anterolateral talar dislocation, no fractures, and enlargement of the tibioperoneal syndesmosis (Fig. 1).

Under locoregional anesthesia, the patient's ankle was manipulated with the purpose of reducing the dislocation. Post-reduction X-rays showed persistent lateral talar subluxation with an asymmetrical joint rim and enlargement of the tibioperoneal syndesmosis (Fig. 2). Because of severe swelling, and in the absence of neurovascular injuries, we decided to postpone the surgical reduction; the patient's right lower limb was put in a cast and in elevation in order to decrease the swelling.

Open reduction was performed after 6 days. A medial skin incision was performed to expose the tibiotalar joint, and this immediately revealed the presence of

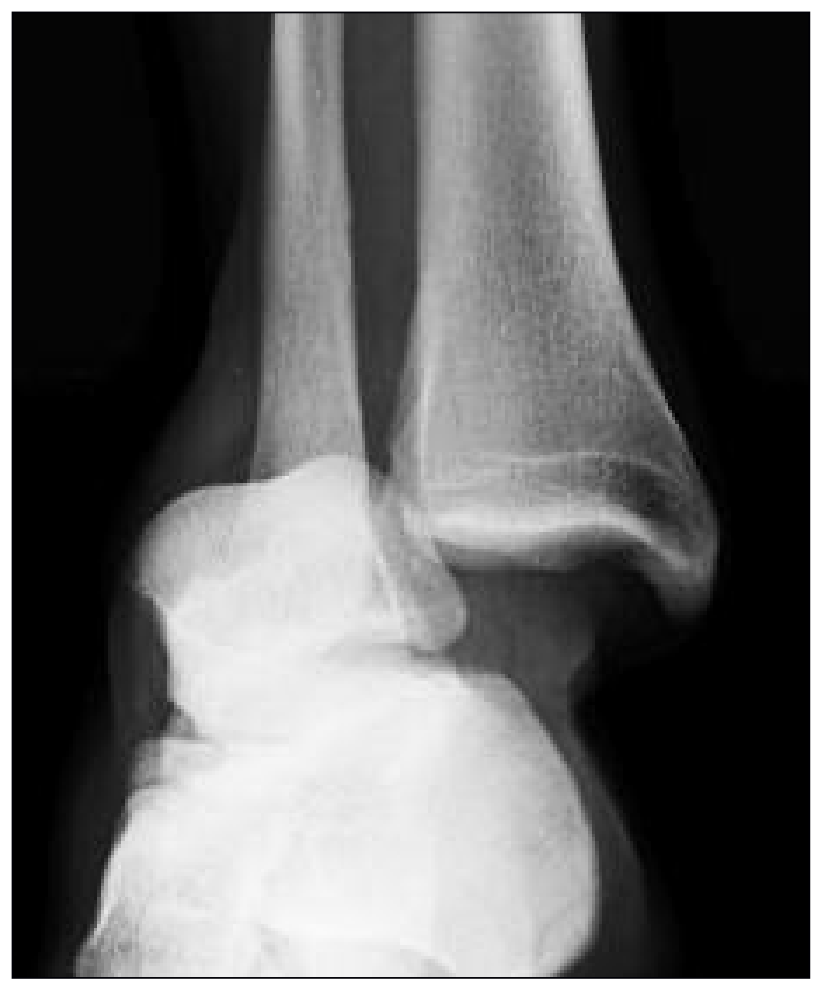

Fig. 1. Standard AP views showing an isolated anterolateral talar dislocation, no fractures, and enlargement of the tibioperoneal syndesmosis.

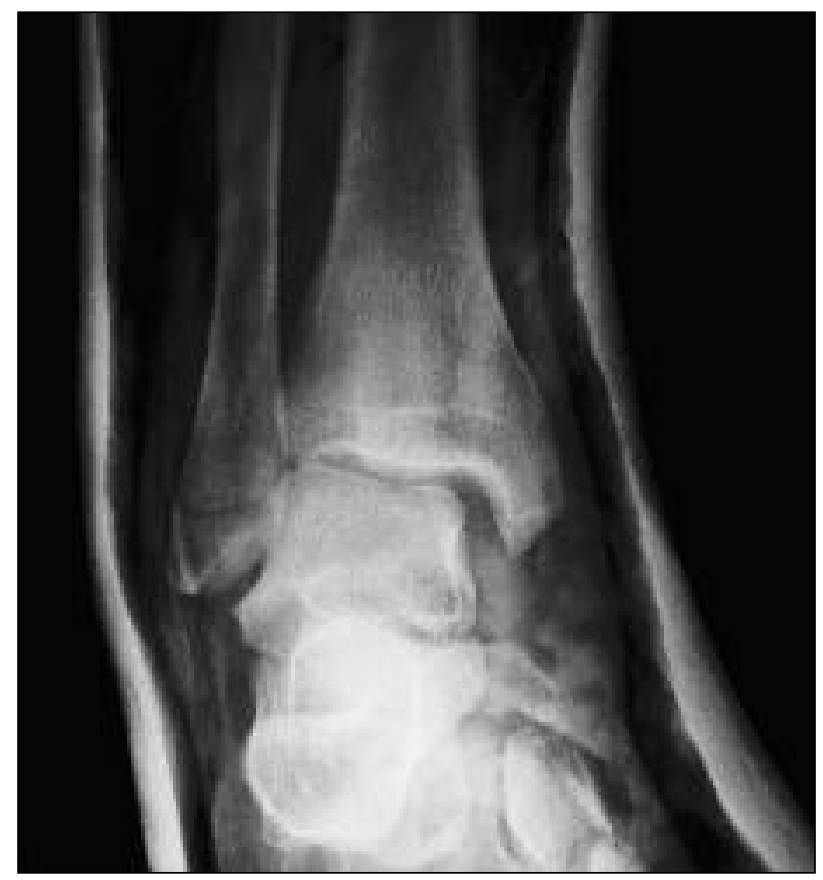

Fig. 2. Post-reduction $\mathrm{X}$-rays showed a persistent lateral talar subluxation with an asymmetrical joint rim and enlargement of the tibioperoneal syndesmosis.

complete soft tissue disruption. This disruption (of the joint capsule, distal tibioperoneal syndesmosis, flexor retinaculum and ligaments) had provoked posterior tibialis tendon dislocation. Unusually, the tendon was not displaced anteriorly to the distal tibia over the medial malleolus, but rather posteriorly, passing through the tibioperoneal syndesmosis to reach the anterior part of the ankle joint, causing lateral traction on the talus; this condition precluded the possibility of performing closed reduction of the ankle joint (Fig. 3). The dislocated posterior tibialis tendon was gently relocated to its normal position in the retromalleolar groove; this maneuver also allowed relocation of the talus in the tibioperoneal mortise (Fig. 4). At this point we were able to proceed with the soft tissue reconstruction: the capsular joint, flexor retinaculum and ligaments were sutured with absorbable stitches (Fig. 5). After the soft tissue reconstruction, the syndesmosis was stabilized with a syndesmotic screw and the tibiotalar joint with a Kirschner wire positioned in a retrograde manner through the heel. After surgery a below-knee cast was applied and no weight bearing was allowed (Fig. 6). 


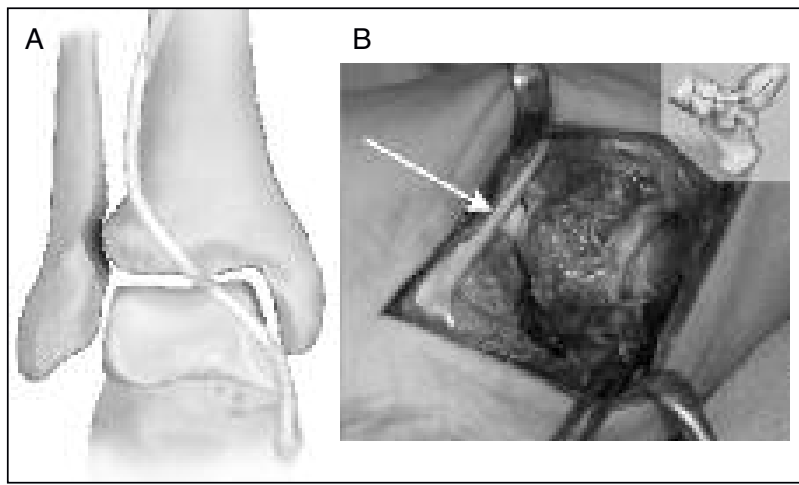

Fig. 3. A: Illustration showing the pattern of the posterior tibialis tendon dislocation. B: The tendon, sliding posteriorly to the distal tibia and passing through the tibioperoneal syndesmosis, reached the anterior part of the ankle joint causing lateral traction on the talus and making it impossible to perform closed reduction of the ankle joint.

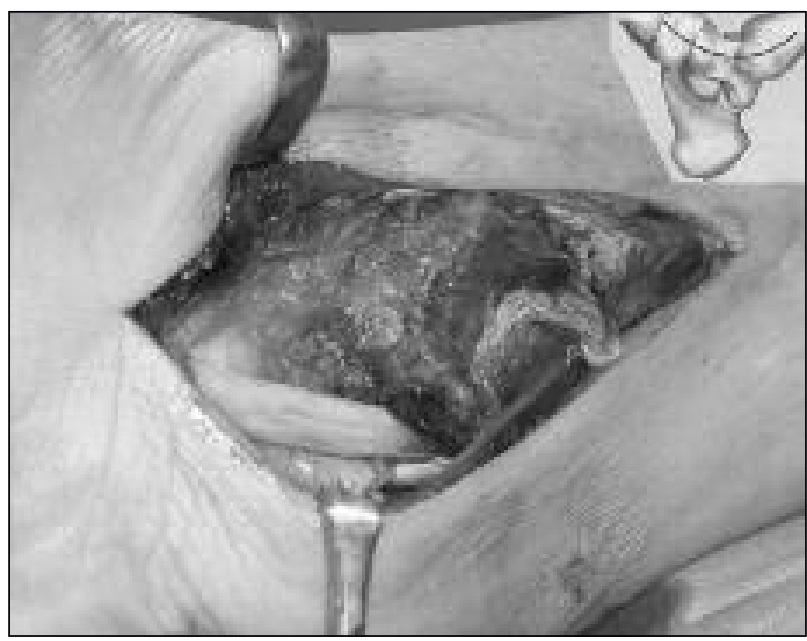

Fig. 4. The dislocated posterior tibialis tendon was gently relocated in the retromalleolar groove.

After 45 days the screw and Kirschner wire were removed and a new below-knee cast was applied. Weight bearing was prohibited for a further month. At 3 months of follow-up, the cast was removed; hydrokinesitherapy was started and partial weight bearing was allowed. X-rays showed maintenance of the reduction of the ankle and tibioperoneal joints (Fig. 7).

At the 4-month follow-up, limitation in dorsiflexion was noted: functional reeducation was continued and

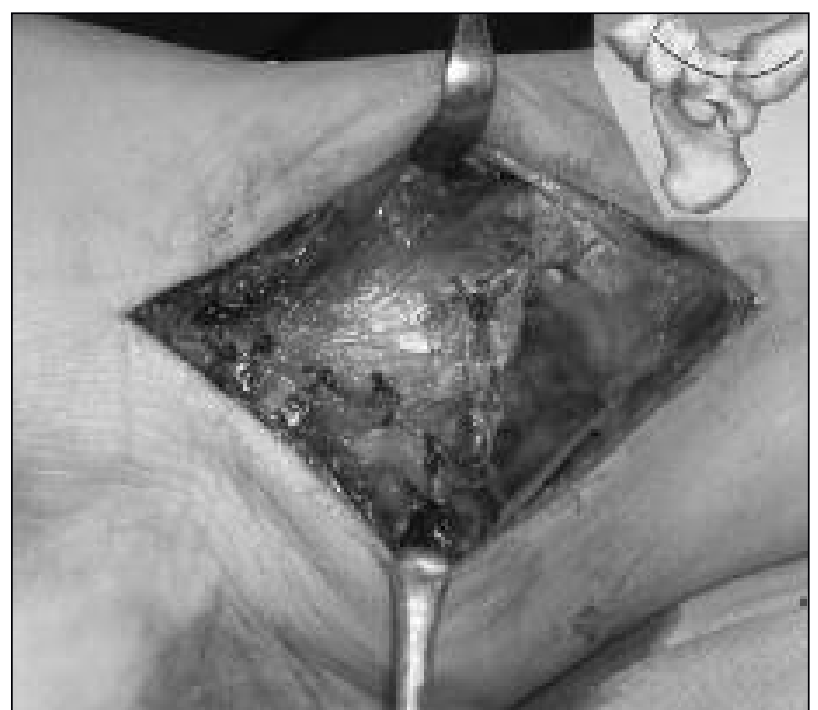

Fig. 5. Soft tissue reconstruction: the capsular joint, flexor retinaculum and ligaments were sutured with absorbable stitches.

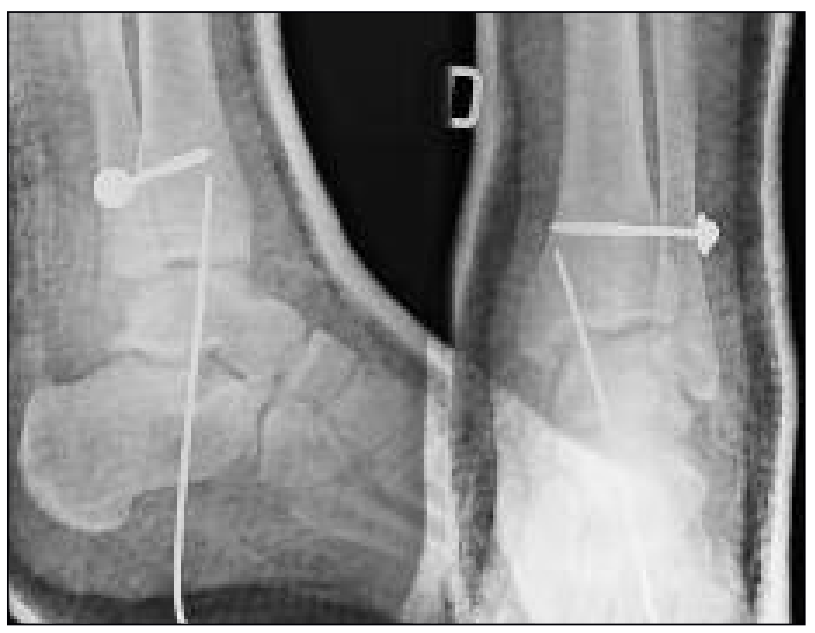

Fig. 6. The syndesmosis was stabilized with a syndesmotic screw and the tibiotalar joint with a Kirschner wire; a below-knee cast was applied.

full weight bearing was allowed. At 18 months the patient returned to our observation with persistent limitation in dorsiflexion and anterior ankle pain during moderate sporting activity. Arthroscopy was performed: anterior synovitis and a small free body were removed; a grade II-III chondral lesion was noted on the medial side of the talar dome and a kissing lesion on the medial surface of the tibia; only debridement was performed. 


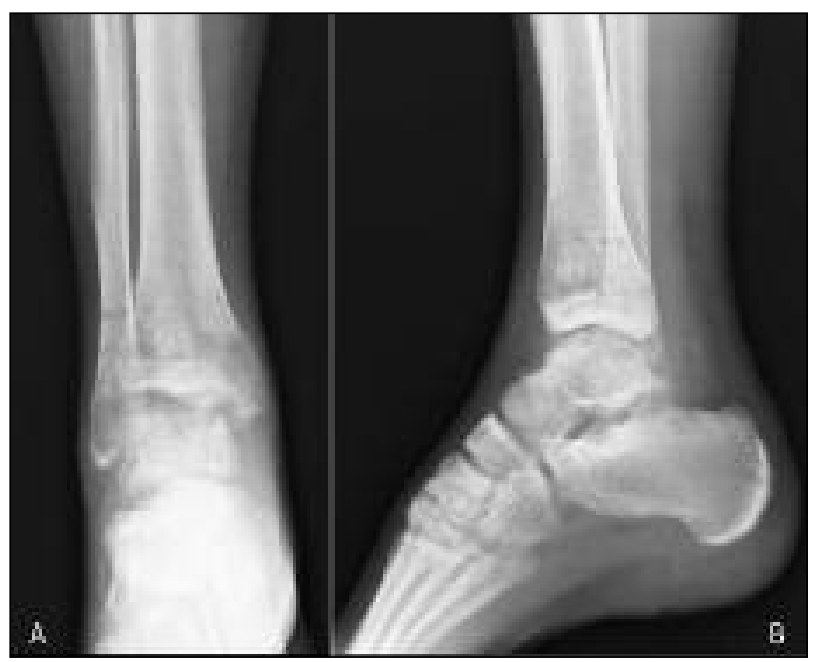

Fig. 7. X-ray taken at 3-month follow-up. A: anteroposterior view. B: lateral view.

\section{Discussion}

In the literature, isolated ankle dislocation is reported in relation to high energy trauma, like a motor vehicle accident or sports injury (the latter especially in situations involving jumping and landing and when the foot is positioned in extreme plantar flexion) (15). Isolated posteromedial dislocation is the type most commonly described (16). This is a rare condition thanks to the highly congruent anatomical configuration of the ankle mortise, in which medial and lateral malleoli greatly reduce the rotational movement of the talus, and the strength of the ligaments higher than the malleoli affords protection against fractures (15). However, other factors, like medial malleolus hypoplasia, laxity of the ligaments, peroneal muscle weakness and previous ankle sprains, could predispose to pure dislocation (17).

When ankle dislocation occurs, the neurovascular condition must be checked before undertaking any attempts at reduction, given that both anterior and posterior neurovascular bundles could be involved in the injury.

Any maneuver should be attempted under local or general anesthesia in order to achieve complete muscle relaxation; when reduction is achieved, a temporary splint should be positioned immediately; indeed, to allow the swelling to subside and to allow better monitoring of the cutaneous and neurovascular con- ditions it is necessary to wait a few days before applying a below-knee cast (16).

If, as in the case described, anatomical reduction cannot be achieved, then the presence of soft tissue interposition should be hypothesized, and if this proves to be the case, open reduction is mandatory. Anterior dislocation of the posterior tibial tendon is quite common and can be caused by forced inversion and dorsiflexion or plantarflexion of the foot during a traumatic injury. The posterior tibial tendon is the most superficial structure in the retromalleolar groove, where it is held in situ by the flexor retinaculum that runs between the tip of the medial malleolus and the calcaneus. Tearing of the fibro-osseous tunnel and flexor retinaculum usually allows the posterior tibial tendon to dislocate anteriorly, passing over the medial malleolus as reported in the literature by several Authors (5,7-11). Other structures that run in the retromalleolar groove (flexor digitorum longus, flexor hallucis longus and posterior neurovascular bundle) are located deeper and are not usually involved in the dislocation. Surgery to treat pure posterior tibialis tendon dislocation has revealed different causes of the dislocation: a flexor retinaculum tear or avulsion, a lax retinaculum, or a shallow retromalleolar groove (18).

In the extremely uncommon case presented here, a high energy trauma caused tibiotalar dislocation with distal tibioperoneal dislocation. This event caused soft tissue disruption, as a result of which the posterior tibialis tendon was able to dislocate itself, unusually, however, it did not pass anteriorly over the medial malleolus, but behind the distal tibia and through the tibioperoneal syndesmosis, placing itself anteriorly between the tibia and talus and making it impossible to achieve reduction of the dislocation, and therefore essential to proceed with open surgery. This path of the posterior tibial tendon dislocation was probably due to an extreme plantarflexion of the ankle joint that caused disruption of the joint capsule, distal tibioperoneal syndesmosis, flexor retinaculum and ligaments, and placed the posterior tibial tendon under tension; it was this tensioning that caused the tendon to slide behind the distal tibia and not anteriorly over the medial malleolus. In 2010, Ermis et al. (12) reported this same pattern of posterior tibial tendon dislocation and interpositioning of the tendon between the distal tibia and fibula in a tibiotalar fracture-dislocation. Trividi et al. (13) reported a posterior tibial ten- 
don dislocation behind the tibia and through the syndesmosis in a case with lateral displacement of the talus and proximal peroneal fracture (Maissoneuve fracture/dislocation): they performed open reduction and screw stabilization of the syndesmosis, which appeared incompletely closed on post-operative Xrays; a CT scan was taken and the tendon dislocation could be noted, therefore another surgery was performed to remove the screw, relocate the posterior tibial tendon, and re-stabilize the syndesmosis. Another similar case of closed fracture-dislocation of the ankle was reported by Lacasse et al. (14) in 2014: after a first surgery for reduction and internal fixation, enlargement of the syndesmosis was still present, therefore revision of the fixation was performed without, however, resulting in successful syndesmosis reduction; only after an MRI was it noted that the posterior tibial tendon was dislocated, entering the ankle joint through the syndesmosis, and hooked underneath the posterior malleolus; a third surgery was performed to relocate the posterior tibial tendon and reduce the syndesmosis.

The pathway of posterior tibial tendon dislocation in the present case was noticed only during surgery, given that, after the unsuccessful reduction maneuver, no instrumental investigations, such as MRI and/or CT scans, were performed. After critically analyzing the cases mentioned $(13,14)$, which were characterized by initial unsuccessful attempts to obtain good reduction through surgery that was performed in the absence of information from MRI or CT scans, and also our own management of the case presented here, we strongly suggest that further investigations, such as MRI and/or CT scans, should be performed after unsuccessful reduction of an ankle dislocation attempted by means of a closed maneuver under local or general anesthesia.

Complications after isolated ankle dislocation are quite common: in particular a 5 to $10^{\circ}$ reduction in range of motion is described by several Authors (19, 20); post-traumatic osteoarthritis is also frequent (21) with persistent pain or discomfort, especially after increased activity (15), which can necessitate further surgical intervention, like debridement, osteophyte removal, arthroplasty or arthrodesis.

Considering the poor vascularization of the talus, soft tissue trauma in this area can give rise to avascular necrosis, which is a significant issue, liable to impair long-term outcome: according to the observations of Kenwright and Taylor (22), the incidence of avascular necrosis of the talus is greater when the talus is markedly displaced. The blood supply to the talus is provided by the tarsal canal artery, a branch of the posterior tibial artery, and by the superior neck branches of the dorsalis pedis artery; anastomoses within the talar bones are numerous.

In a complete talar dislocation, like that reported by Schiffer et al. (23), the blood supply is maintained by the abovementioned superior branches through soft tissue attachments; moreover, neovascularization of the talus was demonstrated by magnetic resonance angiography.

In consideration of the above, we suggest that any open reduction, causing additional soft tissue damage, will impair the talar blood supply and increase the incidence of avascular necrosis. Furthermore, injury to nerves is also reported in pure dislocation of the ankle: the tibial nerve, superficial peroneal nerve and sural nerve can be involved, and this may lead to temporary or permanent deficit (24).

Together, this evidence underlines the importance of checking the neurovascular condition before and after any reduction.

\section{Acknowledgements}

The Authors thank Silvia Bassini for executing the illustrations for this article.

\section{References}

1. Hsiao $\mathrm{KC}, \mathrm{Tu} \mathrm{CH}$. Irreducible fracture dislocation of the ankle: report of two cases. J Formos Med Assoc. 1994;93 (Suppl 3):S161-S165.

2. Schatzker J, Johnson RG. Fracture-dislocation of the ankle with anterior dislocation of the fibula. J Trauma. 1983;23: 420-423.

3. Mayer PJ, Evarts CM. Fracture-dislocation of the ankle with posterior entrapment of the fibula behind the tibia. J Bone Joint Surg Am. 1978;60:320-324.

4. Schatzker J, McBroom R, Dzioba R. Irreducible fracture dislocation of the ankle due to posterior dislocation of the fibula. J Trauma. 1977; 17:397-401.

5. Coonrad RW, Bugg EI Jr. Trapping of the posterior tibial tendon and interposition of the soft tissue in severe fractures about the ankle joint. J Bone Joint Surg Am. 1954;36A:744-750.

6. Woodruff MJ, Brown JN, Mountney J. A mechanism for entrapment of the tibialis posterior tendon in lateral subtalar dislocation. Injury. 1996;27:193-194. 


\section{oints}

7. Böhler L. The Treatment of Fractures, English ed. 4, William Wood Publishers, Baltimore. 1936:453.

8. Parrish TF. Fracture-dislocation of the ankle; an unusual cause of failure of reduction: a case report. J Bone Joint Surg Am. 1959;41-A:749-751.

9. Walker RH, Farris C. Irreducible fracture-dislocations of the ankle associated with interposition of the tibialis posterior tendon: case report and review of the literature of a specific ankle fracture syndrome. Clin Orthop Relat Res. 1981;(160): 212-216.

10. Pankovich AM. Fracture-dislocation of the ankle. Trapping of the posteromedial ankle tendons and neurovascular bundle in the tibiofibular interosseous space: a case report. J Trauma. 1976;16:927-929.

11. Anderson JG, Hansen ST. Fracture-dislocation of the ankle with posterior tibial tendon entrapment within the tibiofibular interosseous space: a case report of a late diagnosis. Foot Ankle Int. 1996;17:114-118.

12. Ermis MN, Yagmurlu MF, Kilinc AS, et al. Irreducible fracture dislocation of the ankle caused by tibialis posterior tendon interposition. J Foot Ankle Surg. 2010;49:166-171.

13. Trividi M, Brown E, Lese A, et al. Lateral dislocation and incarceration of the posterior tibial tendon through the distal tibiofibular syndesmosis. Skeletal Radiol. 2014;43:1175-1178.

14. Lacasse JS, Laflamme M, Penner MJ. Irreducible fracture-dislocation of the ankle associated with interposition of the tibialis posterior tendon in the syndesmosis: a case report. J Foot Ankle Surg. 2015;54:962-966.

15. Georgilas I, Mouzopoulos G. Anterior ankle dislocation without associated fracture: a case with an 11 year follow-up. Acta Orthop Belg. 2008;74:266-269.

16. Ríos-Luna A, Villanueva-Martínez M, Fahandezh-Saddi H, et al. Isolated dislocation of the ankle: two cases and review of the literature. Eur J Orthop Surg Traumatol. 2007;17:403407.

17. Moehring HD, Tan RT, Marder RA, et al. Ankle dislocation. J Orthop Trauma. 1994;8:167-172.

18. Goucher NR, Coughlin MJ, Kristensen RM. Dislocation of the posterior tibial tendon: a literature review and presentation of two cases. Iowa Orthop J. 2006;26:122-126.

19. Toohey JS, Worsing RA Jr. A long-term follow-up study of tibiotalar dislocations without associated fractures. Clin Orthop Relat Res. 1989;(239):207-210.

20. Wroble RR, Nepola JV, Malvitz TA. Ankle dislocation without fracture. Foot Ankle. 1988;9:64-74.

21. Xarchas KC, Psillakis IG, Kazakos KJ, et al. Total dislocation of the talus without a fracture. Open or closed treatment? Report of two cases and review of the literature. Open Orthop J. 2009;3:52-55.

22. Kenwright J, Taylor RG. Major injuries of the talus. J Bone Joint Surg Br. 1970;52:36-48.

23. Schiffer G, Jubel A, Elsner A, et al. Complete talar dislocation without late osteonecrosis: clinical case and anatomic study. J Foot Ankle Surg. 2007;46:120-123.

24. Rivera F, Bertone C, De Martino M, et al. Pure dislocation of the ankle: three case reports and literature review. Clin Orthop Relat Res. 2001;(382):179-184. 